\title{
Luminescence and Morphological Kinetics of Functionalized ZnS Colloidal Nanocrystals
}

\author{
Prabha Sana, Lubna Hashmi, and M. M. Malik \\ Research Laboratory, Department of Physics, Maulana Azad National Institute of Technology, \\ Bhopal 462051, India \\ Correspondence should be addressed to Prabha Sana, prabhasana@yahoo.co.in
}

Received 22 November 2011; Accepted 10 January 2012

Academic Editors: A. S. Gouveia-Neto, J. Jeong, A. V. Kir'yanov, D. Poitras, and J. S. Sanghera

Copyright (๑) 2012 Prabha Sana et al. This is an open access article distributed under the Creative Commons Attribution License, which permits unrestricted use, distribution, and reproduction in any medium, provided the original work is properly cited.

\begin{abstract}
This paper reports functionalized zinc sulphide ( $\mathrm{ZnS}$ ) semiconductor nanocrystals (quantum dots, approx., $2.5 \mathrm{~nm}$ ) which are an important building block in self-assembled nanostructures. $\mathrm{ZnS}$ is functionalized by organic stabilizer Thio glycolic acid (TGA). The samples have been synthesized by colloidal technique at relatively low temperature (below $100^{\circ} \mathrm{C}$ ) at an atmospheric pressure of $10^{-3}$ torr. Manganese (Mn) doping ions have been incorporated (doped) in ZnS host lattice and observed its effect on growth morphology and optical properties of ZnS colloidal nanocrystals. By XRD, SEM, TEM, and PL, the obtained cubic phase nanosized TGA-capped $\mathrm{ZnS}$ materials were characterized. The morphology of $\mathrm{ZnS}$ obtained at different temperatures are analyzed by SEM. The crystallite size of the ZnS nanoparticles was estimated from the X-ray diffraction pattern by using Scherrer's formula (approximately $2.5 \mathrm{~nm}$ ) which is confirmed by TEM. The estimated bandgap value of ZnS NC's by ( $\alpha h v)^{2}$ versus hv́ plot was $4.89 \mathrm{eV}$. Gaussian fitting curve in photoluminescence (PL) spectra indicated room temperature emission wavelength range from 300 to $500 \mathrm{~nm}$ in undoped and Mn-doped ZnS, with different emission peak intensities, and suggested the wide band emission colours in visible and near UV region which has wider applications in optical devices.
\end{abstract}

\section{Introduction}

Zinc sulfide (ZnS), group II-VI compound semiconductor, is having great interest for its practical applications in optoelectronics and photonics [1-9]. Because of its wide bandgap $(3.73 \mathrm{eV})$, it has a high index of reflection and a high transmittance in the visible range, particularly suitable for host material for a large variety of dopants. It has been extensively studied for a variety of applications like optical coatings, field effect transistors, optical sensors, electroluminescence, phosphors, and other light emitting materials [10-12]. Photoluminescence properties of $\mathrm{ZnS}$ have received special attention because of emission in different visible bands due to dopant ions in host matrices. The blue luminescence of $\mathrm{ZnS}$ host and orange luminescence due to the ${ }^{4} \mathrm{~T}_{1}{ }^{-6} \mathrm{~A}_{1}$ transition of $\mathrm{Mn}^{2+}$ ions excited via energy transfer from the host $\mathrm{ZnS}$ have been reported by various researchers $[13,14]$. At nanoregime, the potential advantages have been occurred in $\mathrm{ZnS}$ due to the spatial confinement of photoexcited electrons and holes that modify its optical and other related properties. Many research works have been carried out on these materials at nanoscale $[15,16]$. A variety of methods have been reported for the preparation of nano-ZnS. These methods include soft solution synthesis, sol-gel synthesis, chemical vapor deposition, hydrothermal conditions, microwave irradiation, and so forth [17-27]. These novel routes for synthesis of optoelectronic materials are an integral aspect of material chemistry and physics. The colloidal synthesis route is another novel method of synthesis and is a developing area in the field of research. In this technique, concentration of reagent, capping agent to precursor ratio, $\mathrm{pH}$, time, and temperature play important role to control the morphology and size of the $\mathrm{ZnS}$ nanoparticle. The stoichiometric molar ratio of sodium sulfide to zinc acetate is favorable to produce the cubic $\mathrm{ZnS}$ phase with fine nanoparticles. In the present context, the colloidal technique has been successfully employed for the preparation of TGA- (Thio-glycolic acid-) capped manganese-doped and undoped $\mathrm{ZnS}$ nanomaterials. This paper reports the ZnS QD's of size approximately $2.5 \mathrm{~nm}$ 
with varying morphologies, depending on the concentration of dopant ion and temperature. The photoluminescence emission spectra of TGA-capped manganese- $\left(\mathrm{Mn}^{2+}-\right)$ doped and undoped $\mathrm{ZnS}$ colloidal nanocrystals suggests the wide band emission colours in visible and near UV region.

\section{Experimental Details}

2.1. Synthesis of ZnS Nanoparticles. The chemicals, thio glycolic acid (TGA), zinc acetate, and sodium sulfide obtained from Aldrich and Merck chemicals. For preparation of TGA-capped ZnS, $100 \mathrm{~mL}$ solution of 10.3 mmole TGA in deionized water has been taken in a three-necked round bottom (RB) flask and stirred upto 1 hour. Afterwards, $100 \mathrm{~mL}$ of 10.26 mmole aqueous solution of zinc acetate $\mathrm{Zn}\left(\mathrm{CH}_{3} \mathrm{COO}\right)_{2}$ was added in the TGA solution. The $\mathrm{pH}$ of resultant solution was maintained up to 11.5 in addition of $5 \mathrm{~mL}$ of $1 \mathrm{~N} \mathrm{NaOH}$ aqueous solution and then the round bottom flask kept on stirring for 12 hours in vacuum atmosphere of relative pressure $10^{-3}$ torr at room temperature. After 12 hours, freshly prepared $100 \mathrm{~mL}$ of a 10.26 mmole aqueous solution of sodium sulfide $\mathrm{Na}_{2} \mathrm{~S}$ was injected slowly in the round bottom flask. This leads to a milky white precipitate in the flask, further stirred 2 hours upto $80^{\circ} \mathrm{C}$.

2.2. Synthesis of Manganese-Doped ZnS Nanoparticles. Same procedure was adopted to prepare Mn-doped TGAcapped $\mathrm{ZnS}$, where $50 \mathrm{~mL}$ aqueous solution of $1.03 \mathrm{mmole}$ $\mathrm{Mn}\left(\mathrm{CH}_{3} \mathrm{COO}\right)_{2}$ was added in RB flask. Further, the same procedure was adopted as mentioned in Section 2.1. To observe the temperature and time effect on growth morphology of $\mathrm{ZnS}$, aliquots at temperature $40^{\circ} \mathrm{C}$ and $80^{\circ} \mathrm{C}$ were taken. Subsequently, the prepared samples were centrifuged at $5000 \mathrm{rpm}$ and washed several times with deionized water, later precipitates were collected for further characterization.

2.3. Morphological and Optical Characterization. The Mn incorporated $\mathrm{ZnS}$ was observed by transmission electron microscopy (TEM) (TECNAI-20 $\mathrm{G}^{2}$ ) at $200 \mathrm{kV}$ and the morphology of the Mn-doped $\mathrm{ZnS}$ nanostructures was observed using scanning electron microscope (SEM) JEOL/EO Version-1 model JSM-6390 using stable suspension. The X-ray diffraction patterns of different samples were recorded using a Rigaku Mini Flex 2 Desktop using Kb filter $\mathrm{CuK} \alpha(\lambda=1.54148 \AA)$ radiation at $15 \mathrm{~mA}$ and $30 \mathrm{kV}$ X-ray diffractometer. Room temperature UV-Visible absorption spectra were obtained by UV/Vis spectrophotometer model number 117 Systronics with wavelength range $200 \mathrm{~nm}$ to $800 \mathrm{~nm}$, photoluminescence spectra were studies with LS-55, Perkin Elmer Photoluminescence Spectrophotometer.

\section{Results and Discussions}

3.1. Growth Mechanism of ZnS. Zinc acetate dissociates into zinc ions $\left(\mathrm{Zn}^{2+}\right)$ and acetate $\left(\mathrm{Ac}^{-}\right)$ions in aqueous solution. Similarly, manganese acetate and sodium sulfide dissociate into their respective cation and anions. Sodium being more reactive than zinc and manganese readily forms sodium

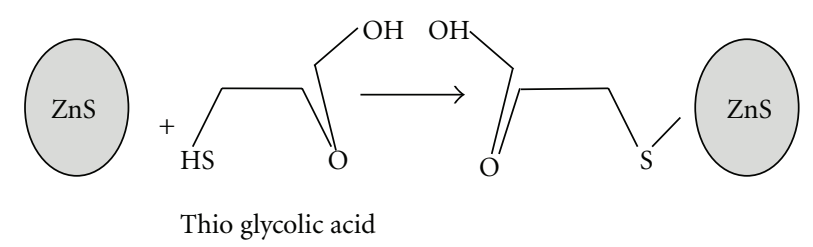

FIGURE 1: Schematic representation of stabilization of $\mathrm{ZnS}$ nanoparticles by thio glycolic acid (TGA).

acetate. $\mathrm{ZnS}$ nuclei form due to the reaction between $\mathrm{Zn}^{2+}$ and $\mathrm{S}^{2-}$ ions, which subsequently grow by consuming more ions from the solution. To prevent further agglomeration to control the size, a repulsive force must be added between the particles to balance the attractive force. This is achieved by adsorbing organic molecules over nanoparticles inducing steric hindrance by employing TGA as the stabilizing agent. Thiol group of TGA gets attached to the surface of $\mathrm{ZnS}$ nanoparticles and prevents further agglomeration to control the size of nanoparticle, shown in Figure 1.

3.2. Structural Analysis. Structural analysis was made on the TGA-capped ZnS nanomaterial by colloidal synthesis process. The X-ray diffractogram in Figures 2(a) and 2(b) indicated the polycrystalline nature of both undoped and Mndoped ZnS materials. The prominent peaks corresponding to (111), (220), and (311) plane reflected to ZnS, which were observed at $2 \theta$ value $28.8,48.0$, and 56.9 , respectively, agreed with JCPDS Card number 80-0020. It reveals face-centered cubic ZnS having zinc blend structure with preferential orientation in the (111) direction. The lattice parameter was estimated and the values of " $a$ " was 5.40 [28]. The XRD peaks are broadened due to nanocrystalline nature of particles because nanocrystals have lesser lattice planes compared to bulk, which contributes to the broadening of the peaks in the diffraction pattern $[29,30]$. From the width of the XRD peak broadening, the mean crystalline size was calculated using Scherrer's equation: $D=0.9 \lambda / \beta \cos \theta$, where $\lambda$ is the X-ray wavelength $(\mathrm{Cu} \mathrm{K \alpha}$ radiation $1.541 \AA), \theta$ is the diffraction angle, and $\beta$ is the half-width of the diffraction peak. The mean crystal sizes of doped and undoped $\mathrm{ZnS}$ nanoparticles are calculated to be $2.5 \mathrm{~nm}$. This is consistent with the estimated size of nanocrystals in TEM images. No apparent difference is observed in the XRD peak shape and broadening of $\mathrm{ZnS}$ nanoparticles before and after $\mathrm{Mn}^{2+}$ doping. The electron microscope studies have been made for TGA-capped $\mathrm{Mn}^{2+}$-doped $\mathrm{ZnS}$ nanoparticles, shown in Figure 3(a). The nano-ZnS fringes are observed clearly and comparable with the XRD data. The electron diffraction pattern of nano$\mathrm{ZnS}$ shows diffraction rings assigned to (111), (220), and (311) planes. In the TEM images, Figure 3(b), the shape of these nanoparticles is spherical. The average size of the nanoparticle is in the range of $2-3 \mathrm{~nm}$, which is also a supportive data for this research and comparable with XRD result.

3.3. Growth Mechanism of Undoped ZnS Nanocrystals. Growth process of nanostructures or microstructures is 


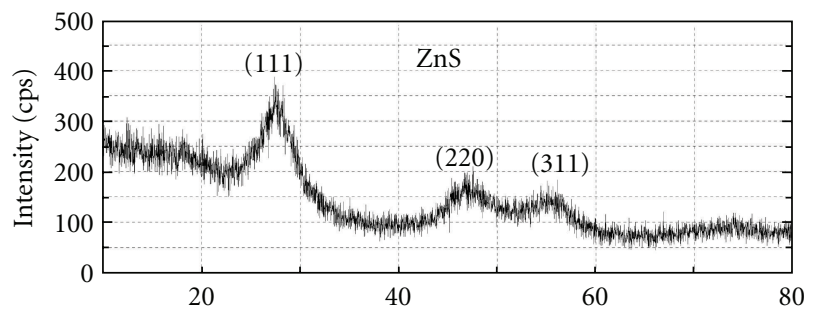

(a)

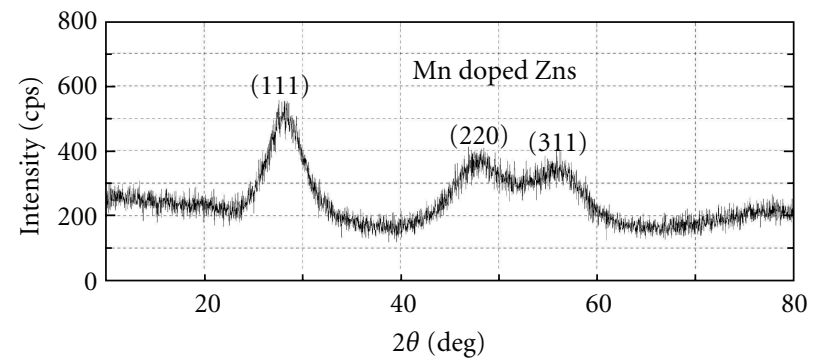

(b)

FIgURE 2: X-Ray diffraction patterns: (a) ZnS, (b) doped ZnS.

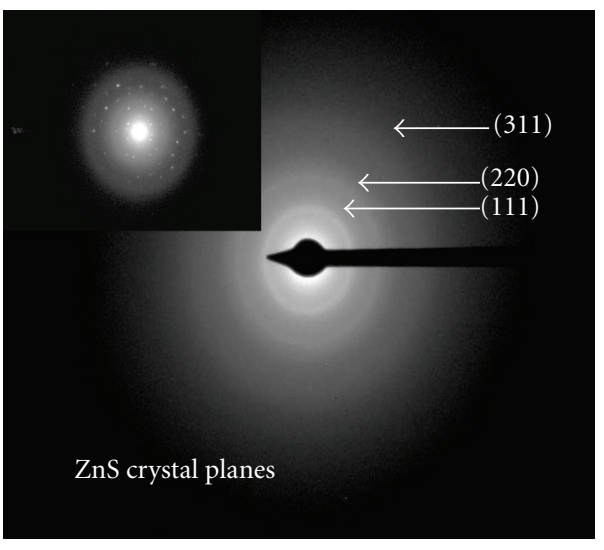

(a)

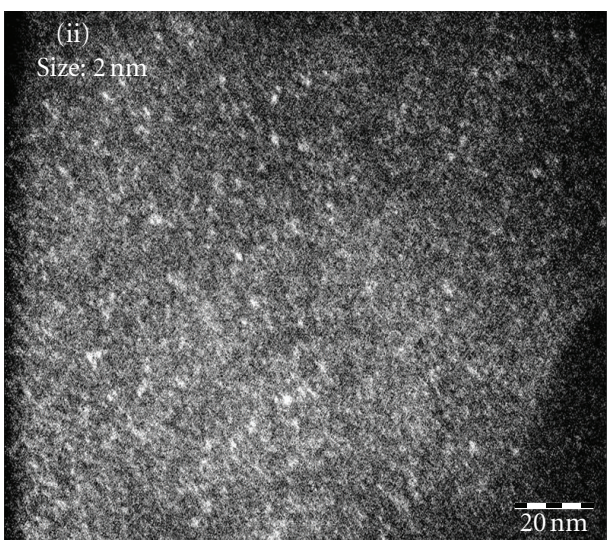

(b)

Figure 3: TEM images of doped ZnS nanoparticles: (a) bright field image with diffraction pattern (b) dark field image.

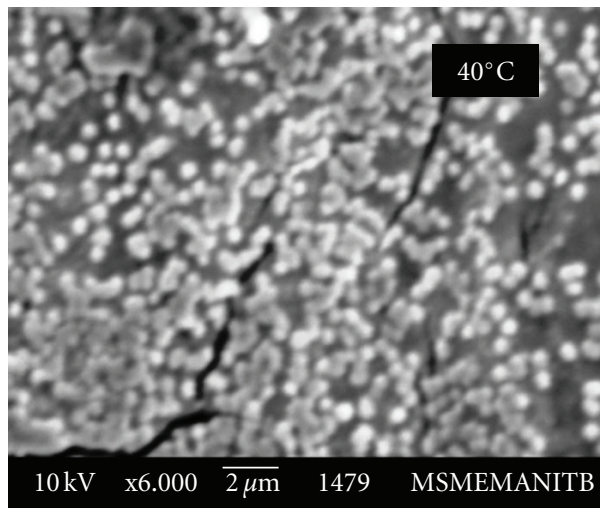

(a)

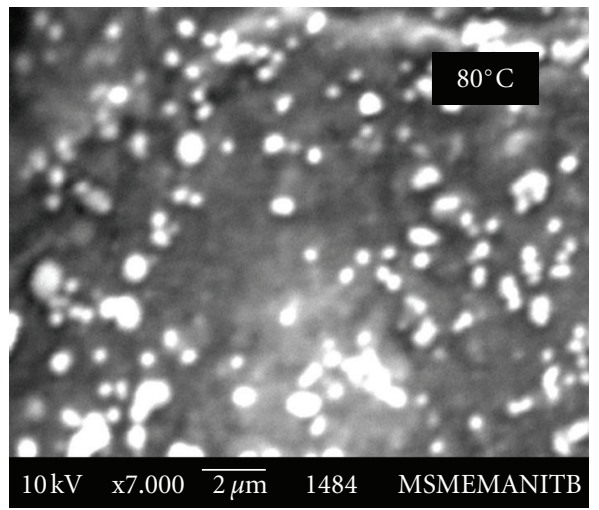

(b)

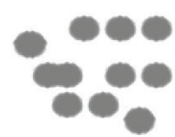

Ostwald ripening

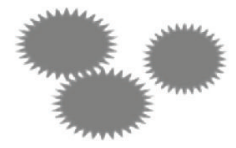

(c)

Figure 4: SEM image TGA-capped ZnS; (a) at $40^{\circ} \mathrm{C}$ and (b) $80^{\circ} \mathrm{C}$. 


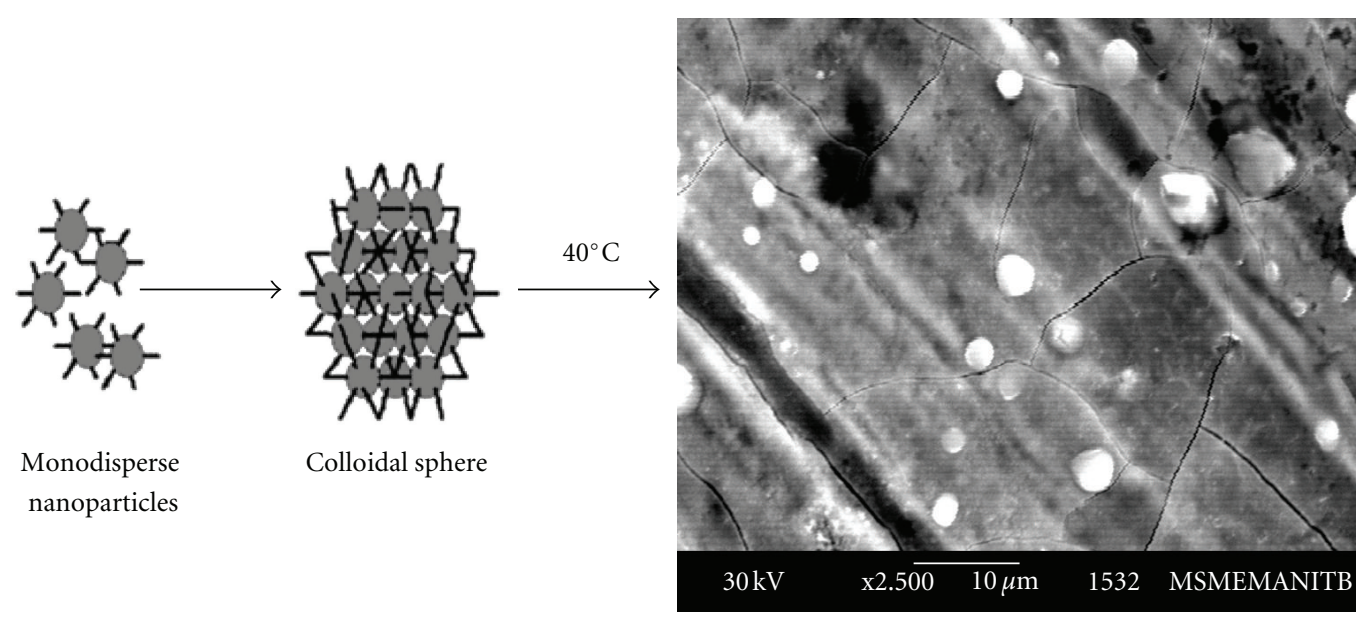

(a)

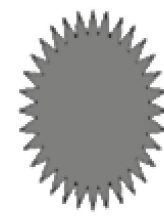

Colloidal sphere

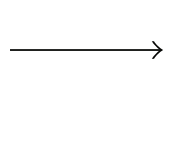

Growth of nanorods

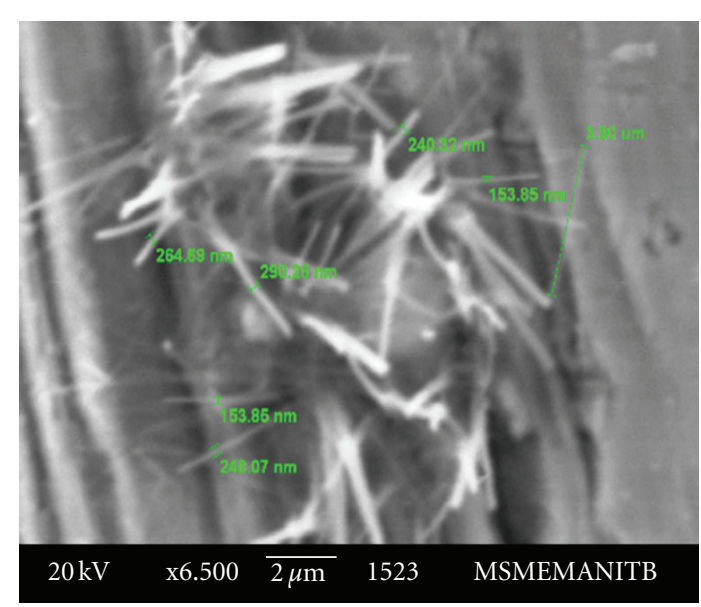

(b)

FIgure 5: SEM micrograph of doped ZnS; (a) at $40^{\circ} \mathrm{C}$ and (b) $80^{\circ} \mathrm{C}$.

basically depending on nanoparticle aggregation. The aggregation of nanoparticles may provide zero, one, two, or three dimensional structures of various shapes. The kinetics of aggregation depends on the growth rate of nanoparticles in the solution and ions concentration. In our case, the aggregation of $\mathrm{ZnS}$ nanoparticles of initial size $2.5 \mathrm{~nm}$ (by TEM analysis) provides the spherical-shaped morphology of size 300 to $500 \mathrm{~nm}$. Morphological analysis of aggregated nanoparticles of TGA-capped undoped ZnS (synthesized at $40^{\circ} \mathrm{C}$ ) is presented in SEM micrograph Figure $4(\mathrm{a})$. The as prepared undoped $\mathrm{ZnS}$ sample shows size distribution approximately $300 \mathrm{~nm}$ to $500 \mathrm{~nm}$. With increased synthesis temperature $80^{\circ} \mathrm{C}$, the sizes of $\mathrm{ZnS}$ nanostructure have been increased, it became approximately 400 to $800 \mathrm{~nm}$ shown in Figure 4(b). The size variations of spherical nanostructures with increased time and temperature is clearly observed. Schematic representation of the growth process of $\mathrm{ZnS}$ spherical nanostructure is shown in Figure 4. This growth process is based on Ostwald repining [31], which suggests that nanocrystal size increases with increasing reaction time and temperature because when the free atoms in solution are supersaturated, the free atoms have a tendency to condense on the surface of larger particles. Therefore, all smaller particles shrink, while larger particles grow, and overall the average size increase.

3.4. Growth Mechanism of Doped ZnS Nanocrystals. Morphological analysis by SEM of TGA-capped manganesedoped $\mathrm{ZnS}$ nanoparticles has been shown in Figure 5 at two synthesized temperature $40^{\circ} \mathrm{C}$ and $80^{\circ} \mathrm{C}$. SEM micrograph shows colloidal microsphere of size 1 to $3 \mu \mathrm{m}$, synthesized at $40^{\circ} \mathrm{C}$ temperature. These colloidal microspheres are selfassembly of nanoparticles. Schematic representation of selfassembly of Mn-doped ZnS nanoparticles into colloidal sphere with SEM micrograph is shown in Figure 5(a). As the synthesis temperature increased, the colloidal sphere started to disappear and growths of one dimensional nanorod have been observed on the surface of colloidal sphere. Schematic representations of growth of one-dimensional nanorods from colloidal sphere and SEM micrograph are shown in Figure 5(b). SEM image shows the approximately $4 \mu \mathrm{m}$ length and $200 \mathrm{~nm}$ diameter of Mn-doped ZnS nanorods. The growth process of colloidal sphere to $1 \mathrm{D}$ structure was demonstrated by Botsan's, Denk and Chua in 1972 [32]. 


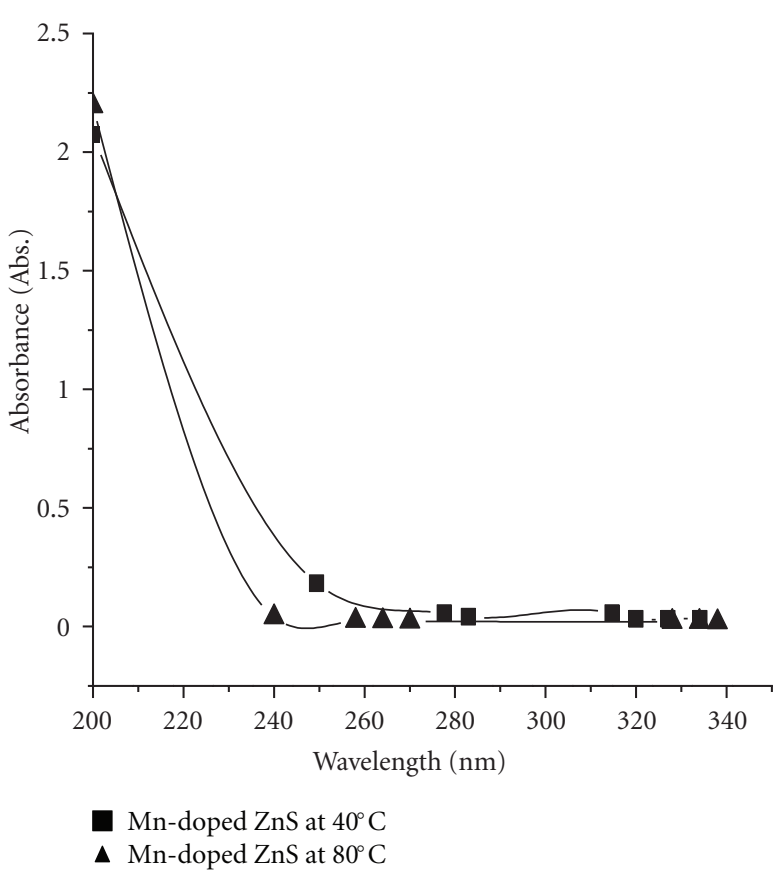

(a)

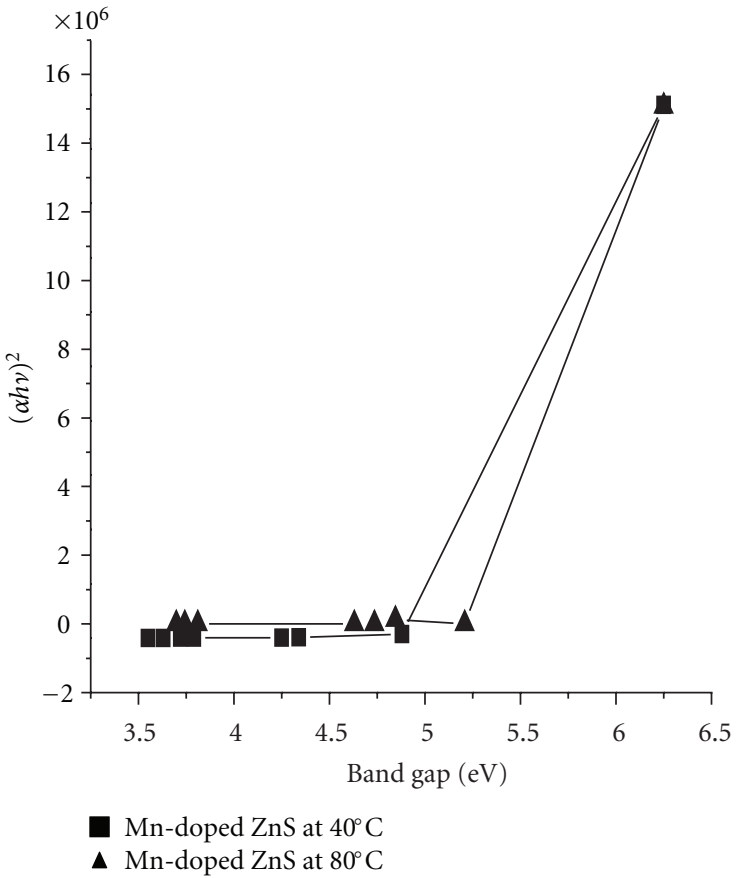

(b)

FIgURE 6: (a) UV-visible absorption graph, (b) $h v$ versus $(\alpha h v)^{2}$ plot.

They suggested the possibility of presence of impurity ions on the surface of colloidal sphere, which could enhance secondary nucleation by adsorbing at defect on existing crystal surface. By initiating crack propagation, crystal prone to disintegration and secondary nucleation may take place, the growth occurs from the impurity ion which is present on the surface. As the time and temperature increase the growth of secondary nucleation increases, the colloidal sphere simultaneously starts to disintegrate. The morphology transformation of colloidal sphere into one-dimensional nanorods of as prepared $\mathrm{Mn}^{2+}$-doped TGA-capped $\mathrm{ZnS}$ material sample has been observed. The impurity ion $\mathrm{Mn}^{2+}$ adsorbed at defect on existing $\mathrm{ZnS}$ crystal surface, responsible for the one-dimensional growth, and also diffused in the interstitial site of $\mathrm{ZnS}$ which modified the optical emission spectra of $\mathrm{ZnS}$ material. Therefore, the doping ion (manganese) has greatly affected the growth of nanostructures as well as their optical properties.

3.5. The Quantum Size Effect and Bandgap Calculation. The UV-visible absorption spectra of $\mathrm{Mn}^{2+}$-doped $\mathrm{ZnS}$ nanoparticles in Figure $6(\mathrm{a})$, at $40^{\circ} \mathrm{C}$ and $80^{\circ} \mathrm{C}$, exhibit absorption band edge at 235 and $253 \mathrm{~nm}$, respectively, resulting from quantum confinement effect. It suggests large blue shift of the absorption from that of $320 \mathrm{~nm}(3.73 \mathrm{eV})$ for bulk $\mathrm{ZnS}$ crystals at room temperature $[33,34]$. Thus, the bandgap of doped $\mathrm{ZnS}$ has been enlarged. Since the crystallite size of doped $\mathrm{ZnS}$ nanoparticles is approximately similar to Bohr radius of exciton in bulk $\mathrm{ZnS}$ crystal, a quantum confinement effect is expected to occur within these crystals, exhibiting an enlargement of the optical bandgap. The optical bandgap has been estimated by $(\alpha h)^{2}$ versus hvi plots, where the optical absorption coefficients $(\alpha)$ were calculated using the following equation:

$$
T=(1-R) \exp (-\alpha t)
$$

where $T$ is transmittance, $R$ is reflectance, and $t$ is the thickness of the sample. The absorption coefficient $(\alpha)$ is related to the incident photon energy as follows:

$$
\alpha h v=k(h v-E g)^{n / 2},
$$

where $k$ is a constant, $E g$ is the optical bandgap of bulk $\mathrm{ZnS}$, and $n$ is a constant equal to 1 for direct bandgap semiconductors and 4 for indirect bandgap materials. Plots of $h v$ versus $(\alpha h v)^{2}$ in Figure 6(b) are linear at the absorption edge, which means that the mode of transition in these nanomaterials has a direct nature. The bandgap energy $E g$ can be obtained from an extrapolation of the straight-line portion of the $(\alpha h v)^{2}$ versus $h v$ plot to zero absorption coefficient value [35]. $(\alpha h v)^{2}$ versus $h v$ plot provides the estimated bandgap $5.2 \mathrm{eV}$ and $4.89 \mathrm{eV}$ for $\mathrm{Mn}^{2+}$-doped $\mathrm{ZnS}$ nanoparticles at synthesized temperatures $40^{\circ} \mathrm{C}$ and $80^{\circ} \mathrm{C}$, respectively. The bandgap energy clearly shifts to a lower wavelength (higher energy) with decreasing synthesis temperature at time. This can be attributed to a quantum confinement effect in nanosize particles. The approximate bandgap $4.9 \mathrm{eV}$ has been estimated for particle size of $2.5 \mathrm{~nm}$ by Bruse equation [36], which is similar to the bandgap calculated by $h \nu$ versus $(\alpha h \nu)^{2}$ plots, proved the direct optical bandgap of as-prepared nano-ZnS. 


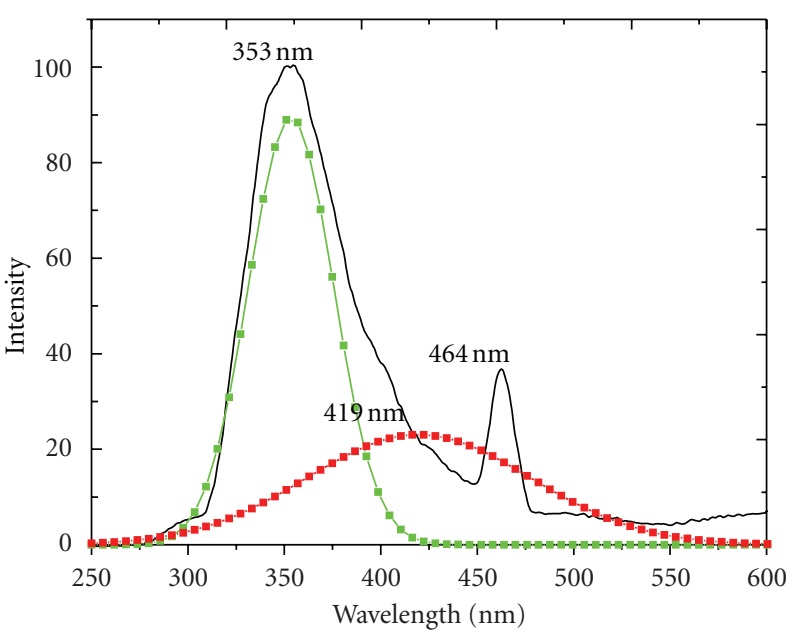

(a)

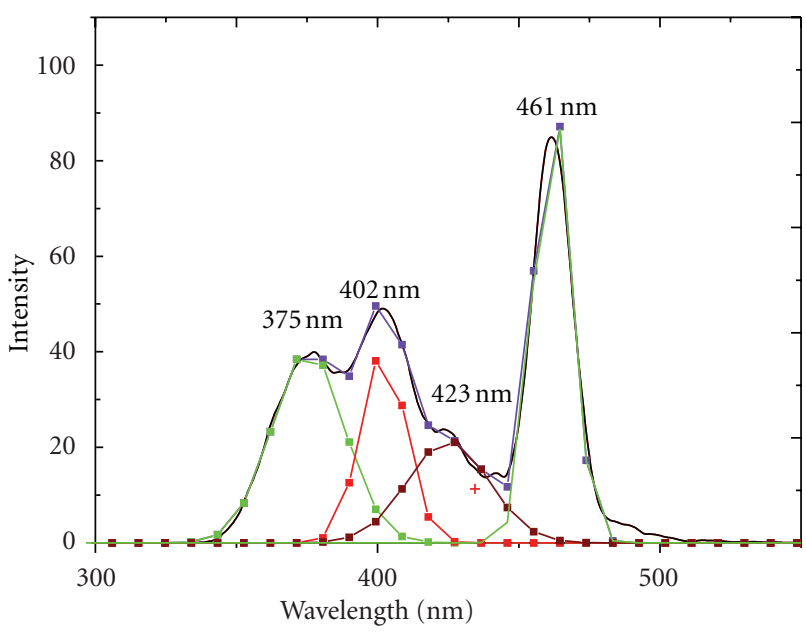

(b)

Figure 7: Photoluminescence (PL) spectrum of (a) undoped ZnS and (b) Mn doped ZnS nanoparticles.

3.6. Emission Spectra Analysis. Room temperature photoluminescence (PL) emission spectra of undoped and Mndoped $\mathrm{ZnS}$ were taken. Doped $\mathrm{ZnS}$ nanoparticles were prepared from a solution with $\mathrm{Mn} / \mathrm{Zn}$ molar ratio 0.01 at $80^{\circ} \mathrm{C}$. The excitation wavelength $250 \mathrm{~nm}(4.9 \mathrm{eV})$ for both doped and undoped sample was blue shifted, from excitation wavelength $332 \mathrm{~nm}(3.73 \mathrm{eV})$ corresponding to the bandgap energy of bulk ZnS host. This blue shift in $\mathrm{ZnS}$ nanocrystals is a reflection of the bandgap increase owing to the quantum confinement effect. Photoluminescence (PL) emission spectrum with Gaussian fitting for undoped $\mathrm{ZnS}$ has been shown in Figure 7(a). Two characteristic bands of undoped ZnS with emission wavelengths at 353 and $464 \mathrm{~nm}$ were observed. PL emission wavelength at $353 \mathrm{~nm}$ (ultraviolet color) contributed to near-band-edge emission originates from the recombination of free excitons of $\mathrm{ZnS}$ nanocrystalline size regime. PL emission peak at 464 should be assigned to stoichiometric vacancies (defect states) or interstitial impurities, possibly at the surface in the $\mathrm{ZnS}$ nanoparticles [37-39]. Gaussian fitting curve with less intense blue emission at $419 \mathrm{~nm}$ is attributed to the sulphur vacancies. The comparative intensity of emission wavelength shows the less interstitial $S^{2-}$ ions, vacancies, and surface defects present in the $\mathrm{ZnS}$ nanocrystals. The photoluminescence emission band at approximately $590 \mathrm{~nm}$ (2.1 eV; yellowish orange) is the characteristic emission of $\mathrm{Mn}^{2+}$ ions in $\mathrm{ZnS}$ crystals, which can be attributed to ${ }^{4} \mathrm{~T}_{1}$ (excited) to ${ }^{6} \mathrm{~A}_{1}$ (ground) transition of the $\mathrm{Mn}^{2+}$ ions in $\mathrm{T}_{d}$ symmetry. Emission occurs through energy transfer from the excited state of the $\mathrm{ZnS}$ host lattice to the d electrons of $\mathrm{Mn}^{2+}[40,41]$. This clearly suggests that the emission at $590 \mathrm{~nm}(2.1 \mathrm{eV})$ arises from $\mathrm{Mn}^{2+}$ ions, incorporated into the $\mathrm{ZnS}$ nanocrystals and not from $\mathrm{Mn}^{2+}$ ions of $\mathrm{MnS}$ nanocrystals. It is important to note that the $\mathrm{Mn}^{2+}$ ions in $\mathrm{MnS}$ nanocrystals have two emission peaks at 1.66 and $1.8 \mathrm{eV}$. Therefore, the presence of $\mathrm{Mn}^{2+}$ emission at $590 \mathrm{~nm}$ indicates that the $\mathrm{Mn}^{2+}$ ions occupy the $\mathrm{Zn}^{2+}$ ion site. More aspects of $\mathrm{Mn}^{2+}$ incorporation in $\mathrm{ZnS}$ host lattice have been made by Kennedy et al. [42] stated that there are two types of $\mathrm{Mn}$ in $\mathrm{ZnS}: \mathrm{Mn}$ nanocrystallites. One is located inside the crystal or very close to another Mn. The other type of $\mathrm{Mn}$ is located at or near the surface. The latter shows the unusual fluorescence of interest. Sooklal et al. [43] reported that $\mathrm{Mn}^{2+}$ incorporated into the $\mathrm{ZnS}$ lattice led to $\mathrm{Mn}^{2+}$-based orange emission while $\mathrm{ZnS}$ with surface-bound $\mathrm{Mn}^{2+}$ yielded ultraviolet emission. In present investigation, emission at $590 \mathrm{~nm}$ due to $\mathrm{Mn}^{2+}$ in $\mathrm{ZnS}$ host lattice is not observed shown Figure $7(\mathrm{~b})$, indicating that the $\mathrm{Mn}^{2+}$ ions were not occupied the $\mathrm{Zn}^{2+}$ ion site. The intensity of the UV emission at $353 \mathrm{~nm}$ which was observed in undoped $\mathrm{ZnS}$ in Figure 7(a) was reduced and this band edge emission shifts in the region $375-461 \mathrm{~nm}$ for Mnincorporated $\mathrm{ZnS}[44,45]$, as shown in Figure 7(b). Hu and Zhang [46] explained that this red-shift may come from the quantum confinement effect in nanoparticles, which leads to the change of the field surrounding the dopant ions [47]. Gaussian fitting for Mn-incorporated $\mathrm{ZnS}$ has been shown in Figure 7(b). Four characteristic bands of Mn:ZnS with emission wavelengths at $375 \mathrm{~nm}, 402 \mathrm{~nm}, 423 \mathrm{~nm}$, and $461 \mathrm{~nm}$ were observed. Emission peaks at $402 \mathrm{~nm}$ and $423 \mathrm{~nm}$ attributed to sulfur vacancies, while surface-bound $\mathrm{Mn}^{2+}$ yielded ultraviolet emission peak at $375 \mathrm{~nm}$, which clearly supports our argument for the morphology variation due to dopant ion. There is another important effect that needs to be observed, that the increased intensity of intense blue emission at $461 \mathrm{~nm}$ in case of doped $\mathrm{ZnS}$ nanoparticle may be confirming that the incorporation of the Mn ions into the $\mathrm{ZnS}$ lattice resulted in activation of surface defect sites and had an effect on the emission as well as growth of $\mathrm{ZnS}$ nanostructures.

\section{Conclusions}

$\mathrm{ZnS}$ and $\mathrm{ZnS}: \mathrm{Mn}$ nanoparticles were prepared using a colloidal synthesis method with thioglycolic acid (TGA) as a surface stabilizing agent to prevent surface dangling 
bonds and agglomeration of in $\mathrm{ZnS}$ nanocrystals. The $\mathrm{ZnS}$ nanocrystal size was controlled by the synthesis temperature. Temperature ranges from $40^{\circ} \mathrm{C}$ to $80^{\circ} \mathrm{C}$ have been used in synthesis process to observe the effect on optical bandgap and morphology of $\mathrm{ZnS}$ nanocrystals. The prepared samples were highly transparent $(>80 \%)$ in the visible region. The absorption edge of doped $\mathrm{ZnS}$ samples was demonstrated narrow grain size distribution. The effect of dopant ions $\mathrm{Mn}^{2+}$ is observed on growth morphology and optical behavior $\mathrm{ZnS}$ nanostructures. It was found that the surface bound $\mathrm{Mn}^{2+}$ ion in $\mathrm{ZnS}$ nanocrystals provides ultraviolet emission. XRD reveals the formation of face-centred cubic $\mathrm{ZnS}$ quantum dots with grains size approximately $2.5 \mathrm{~nm}$ for both undoped and $\mathrm{Mn}$-doped $\mathrm{ZnS}$, this result was confirmed by TEM. These materials show strong emission, are highly transparent in the visible range, and may be useful in fabrication of flat panel display devices.

\section{Acknowledgments}

Thanks are due to the Director, MANIT, Bhopal for providing fellowship and required facilities to P. Sana. Thanks are also due to, Director UGC-DAE Consortium for Scientific Research Centre Indore for providing TEM facility.

\section{References}

[1] Y. Xia, P. Yang, Y. Sun et al., "One-dimensional nanostructures: synthesis, characterization, and applications," Advanced Materials, vol. 15, no. 5, pp. 353-389, 2003.

[2] Z. Tang and N. A. Kotov, "One-dimensional assemblies of nanoparticles: preparation, properties, and promise," Advanced Materials, vol. 17, no. 8, pp. 951-962, 2005.

[3] I. Matsui, "Nanoparticles for electronic device applications: a brief review," Journal of Chemical Engineering of Japan, vol. 38, no. 8, pp. 535-546, 2005.

[4] Y. W. Jun, J. W. Seo, J. O. Sang, and J. Cheon, "Recent advances in the shape control of inorganic nano-building blocks," Coordination Chemistry Reviews, vol. 249, no. 17-18, pp. 1766-1775, 2005.

[5] S. Bhattacharyya, I. Perelshtein, O. Moshe, D. H. Rich, and A. Gedanken, "One-step solvent-free synthesis and characterization of $\mathrm{Zn}_{1-\mathrm{x}} \mathrm{Mn}_{\mathrm{x}} \mathrm{Se} \mathrm{C}$ nanorods and nanowires," Advanced Functional Materials, vol. 18, no. 11, pp. 1641-1653, 2008.

[6] A. Dev, S. Chaudhuri, and B. N. Dev, "ZnO 1-D nanostructures: low temperature synthesis and characterizations," Bulletin of Materials Science, vol. 31, no. 3, pp. 551-559, 2008.

[7] G. Murugadoss, B. Rajamannan, and U. Madhusudhanan, "Synthesis and characterization of water-soluble $\mathrm{ZnS}: \mathrm{Mn}^{2+}$ nanocrystals," Chalcogenide Letters, vol. 6, no. 5, pp. 197-201, 2009.

[8] P. Vinotha Boorana Lakshmi, K. Sakthi Raj, and K. Ramachadran, "Synthesis and characterization of nano $\mathrm{ZnS}$ doped with Mn," Crystal Research and Technology, vol. 44, no. 2, pp. 155158, 2009.

[9] H. Yang, S. Santra, and P. H. Holloway, "Syntheses and applications of Mn-doped II-VI semiconductor nanocrystals," Journal of Nanoscience and Nanotechnology, vol. 5, no. 9, pp. 1364-1375, 2005.
[10] R. Devi, P. Purkayastha, P. K. Kalita, and B. K. Sarma, "Synthesis of nanocrystalline CdS thin films in PVA matrix," Bulletin of Materials Science, vol. 30, no. 2, pp. 123-128, 2007.

[11] I. Moreels, J. C. Martins, and Z. Hens, "Solution NMR techniques for investigating colloidal nanocrystal ligands: a case study on trioctylphosphine oxide at InP quantum dots," Journal of Sensors and Actuators B, vol. 126, no. 1, pp. 283-288, 2007.

[12] G. M. Whitesides and M. Boncheva, "Beyond molecules: self-assembly of mesoscopic and macroscopic components," Proceedings of the National Academy of Sciences of the United States of America, vol. 99, no. 8, pp. 4769-4774, 2002.

[13] K. Jayanthi, S. Chawla, H. Chander, and D. Haranath, "Structural, optical and photoluminescence properties of $\mathrm{ZnS}$ : $\mathrm{Cu}$ nanoparticle thin films as a function of dopant concentration and quantum confinement effect," Crystal Research and Technology, vol. 42, no. 10, pp. 976-982, 2007.

[14] R. N. Bhargava, D. Gallagher, X. Hong, and A. Nurmikko, "Optical properties of manganese-doped nanocrystals of ZnS," Physical Review Letters, vol. 72, no. 3, pp. 416-419, 1994.

[15] J. Zhu, M. Zhou, J. Xu, and X. Liao, "Preparation of CdS and ZnS nanoparticles using microwave irradiation," Materials Letters, vol. 47, no. 1-2, pp. 25-29, 2001.

[16] Y. Ohko, M. Setani, T. Sakata, H. Mori, and H. Yoneyama, "Preparation of monodisperse $\mathrm{ZnS}$ nanoparticles by size selective potocorrosion," Chemistry Letters, no. 7, pp. 663-664, 1999.

[17] L. V. Ruitao, C. Chuanboa, and H. Zhu, "Synthesis and characterization of $\mathrm{ZnS}$ nanowires by AOT micelle-template inducing reaction," Materials Research Bulletin, vol. 39, no. 10, pp. 1517-1524, 2004.

[18] J. Chen, Y. Li, Y. Wang, J. Yun, and D. Cao, "Preparation and characterization of zinc sulfide nanoparticles under highgravity environment," Materials Research Bulletin, vol. 39, no. 2, pp. 185-194, 2004.

[19] H. Chander, V. Shanker, D. Haranath, S. Dudeja, and P. Sharma, "Characterization of $\mathrm{ZnS}: \mathrm{Cu}, \mathrm{Br}$ electroluminescent phosphor prepared by new route," Materials Research Bulletin, vol. 38, no. 2, pp. 279-288, 2003.

[20] B. Vaidhyanathan, M. Ganguli, and K. J. Rao, "Fast solid state synthesis of metal vanadates and chalcogenides using microwave irradiation," Materials Research Bulletin, vol. 30, no. 9, pp. 1173-1177, 1995.

[21] V. G. Bessergenev, N. Ivanova, Yu. A. Kovalevskaya et al., "Optical and structural proper tees of $\mathrm{ZnS}$ and $\mathrm{ZnS}: \mathrm{Mn}$ films prepared by CVD method," Materials Research Bulletin, vol. 30, no. 11, pp. 1393-1400, 1995.

[22] N. I. Kovtyukhova, E. V. Buzaneva, C. C. Waraksa, B. R. Martin, and T. E. Mallouk, "Surface sol-gel synthesis of ultrathin semiconductor films," Chemistry of Materials, vol. 12, no. 2, pp. 383-389, 2000.

[23] K. R. Murali, V. Swaminathan, and D. C. Trivedi, "Characteristics of nanocrystalline CdSe films," Solar Energy Materials and Solar Cells, vol. 81, no. 1, pp. 113-118, 2004.

[24] I. O. Oladeji and L. Chow, "Optimization of chemical bath deposited cadmium sulfide thin films," Journal of the Electrochemical Society, vol. 144, no. 7, pp. 2342-2346, 1997.

[25] B. G. Wang, E. W. Shi, and W. Z. Zhong, "On the morphological changes and twinning of $\mathrm{ZnS}$ (sphalerite) crystallites under hydrothermal conditions," Crystal Research and Technology, vol. 35, no. 3, pp. 279-289, 2000.

[26] A. Vadivel Murugan, R. S. Sonawane, B. B. Kale, S. K. Apte, and A. V. Kulkarni, "Microwave-solvothermal synthesis of 
nanocrystalline cadmium sulfide," Materials Chemistry and Physics, vol. 71, no. 1, pp. 98-102, 2001.

[27] A. Vadivel Murugan, J. Arokia Raj, and V. Swaminathan, "Effect of photoluminescence for CdS nanocrystalline material prepared by microwave solvothermal technique," in Proceedings of the 2nd International Conference on Material for Advanced Technology, NTU, Singapore, December 2003.

[28] W. G. Becker and A. J. Bard, "Photoluminescence and photoinduced oxygen adsorption of colloidal zinc sulfide dispersions," Journal of Physical Chemistry, vol. 87, no. 24, pp. 4888-4893, 1983.

[29] U. K. Gautam, X. Fang, Y. Bando, J. Zhan, and D. Golberg, "Synthesis, structure, and multiple enhanced field-emission properties of branched $\mathrm{ZnS}$ nanotube-in nanowire coreshell heterostructures," ACS Nano, vol. 2, no. 5, pp. 1015-1021, 2008.

[30] S. W. Lu, B. I. Lee, Z. L. Wang et al., "Synthesis and photoluminescence enhancement of $\mathrm{Mn}^{2+}$-doped $\mathrm{ZnS}$ nanocrystals," Journal of Luminescence, vol. 92, no. 1-2, pp. 73-78, 2001.

[31] Y. Yin and A. P. Alivisatos, "Colloidal nanocrystal synthesis and the organic-inorganic interface," Nature, vol. 437, no. 7059, pp. 664-670, 2005.

[32] Y. Hu, Y. Wu, J. Cai et al., "Self-assembly and fractal feature of chitosan and its conjugate with metal ions: $\mathrm{Cu}(\mathrm{II}) / \mathrm{Ag}(\mathrm{I})$," International Journal of Molecular Sciences, vol. 8, no. 1, pp. 112, 2007.

[33] R. N. Bhargava, D. Gallagher, and T. Welker, "Doped nanocrystals of semiconductors-a new class of luminescent materials," Journal of Luminescence, vol. 60-61, pp. 275-280, 1994.

[34] R. N. Bhargava, D. Gallagher, X. Hong, and A. Nurmikko, "Optical properties of manganese-doped nanocrystals of ZnS," Physical Review Letters, vol. 72, no. 3, pp. 416-419, 1994.

[35] A. A. R. Elshabini-Raid and F. D. Barlow, Thin Film Technology Handbook, McGraw-Hill, New York, NY, USA, 1998.

[36] V. L. Klimov, "Nanocrystal quantum dots from fundamental photo physics to multicolour lasing," Los Alamos Science, no. 28, pp. 214-220, 2003.

[37] M. Sharma, S. Kumar, and O. P. Pandey, "Photophysical and morphological studies of organically passivated coreshell ZnS nanoparticles," Digest Journal of Nanomaterials and Biostructures, vol. 3, no. 4, pp. 189-197, 2008.

[38] M. Bruchez, M. Moronne, P. Gin, S. Weiss, and A. P. Alivisatos, "Semiconductor nanocrystals as fluorescent biological labels," Science, vol. 281, no. 5385, pp. 2013-2016, 1998.

[39] D. Denzler, M. Olschewski, and K. Sattler, "Luminescence studies of localized gap states in colloidal ZnS nanocrystals," Journal of Applied Physics, vol. 84, no. 5, pp. 2841-2845, 1998.

[40] L. M. Gan, B. Liu, C. H. Chew et al., "Enhanced photoluminescence and characterization of $\mathrm{Mn}$-doped $\mathrm{ZnS}$ nanocrystallites synthesized in microemulsion," Langmuir, vol. 13, no. 24, pp. 6427-6431, 1997.

[41] I. Yu, T. Isobe, and M. Senna, "Optical properties and characteristics of ZnS nano-particles with homogeneous Mn distribution," Journal of Physics and Chemistry of Solids, vol. 57, no. 4, pp. 373-379, 1996.

[42] T. A. Kennedy, E. R. Glaser, P. B. Klein, and R. N. Bhargava, "Symmetry and electronic structure of the Mn impurity in ZnS nanocrystals," Physical Review B, vol. 52, no. 20, pp. R14356-R14359, 1995.

[43] K. Sooklal, B. S. Cullum, S. M. Angel, and C. J. Murphy, "Photophysical properties of ZnS nanoclusters with spatially localized $\mathrm{Mn}^{2+}$," Journal of Physical Chemistry, vol. 100, no. 11, pp. 4551-4555, 1996.
[44] K. Jayanthi, S. Chawla, H. Chander, and D. Haranath, "Structural, optical and photoluminescence properties of $\mathrm{ZnS}$ : $\mathrm{Cu}$ nanoparticle thin films as a function of dopant concentration and quantum confinement effect," Crystal Research and Technology, vol. 42, no. 10, pp. 976-982, 2007.

[45] R. N. Bhargava, D. Gallagher, X. Hong, and A. Nurmikko, "Optical properties of manganese-doped nanocrystals of $\mathrm{ZnS}$," Physical Review Letters, vol. 72, no. 3, pp. 416-419, 1994.

[46] H. Hu and W. Zhang, "Synthesis and properties of transition metals and rare-earth metals doped ZnS nanoparticles," Optical Materials, vol. 28, no. 5, pp. 536-550, 2006.

[47] D. Sridevi and K. V. Rajendran, "Enhanced photoluminescence of $\mathrm{ZnS}$ nanoparticles doped with transition and rare earth metallic ions," Chalcogenide Letters, vol. 7, no. 6, pp. 397401,2010 . 

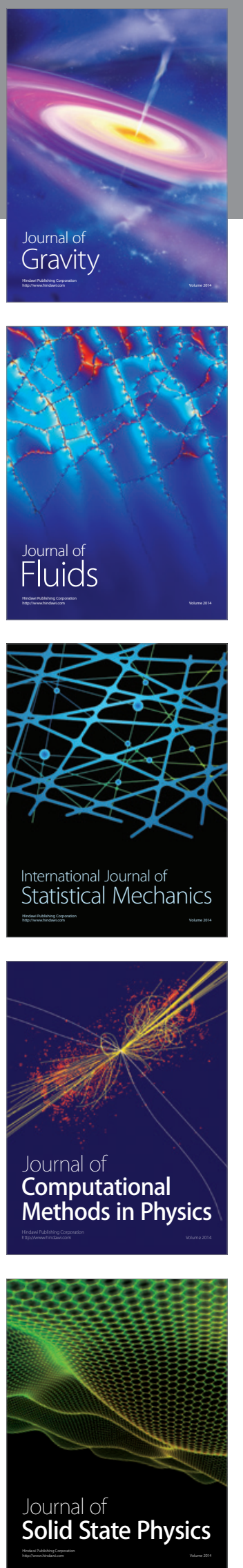
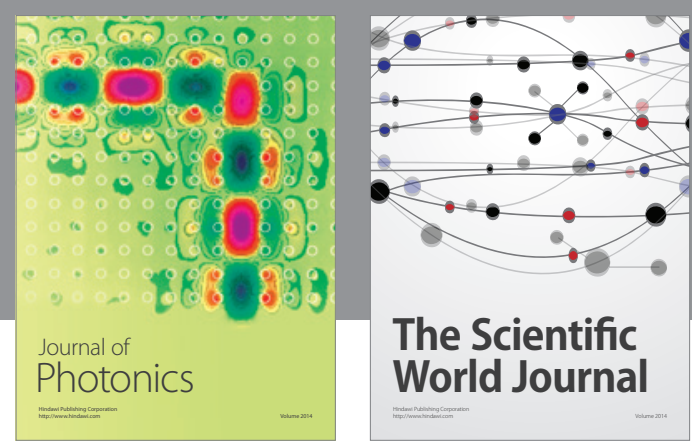

The Scientific World Journal

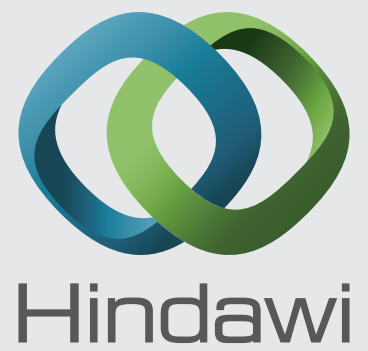

Submit your manuscripts at http://www.hindawi.com
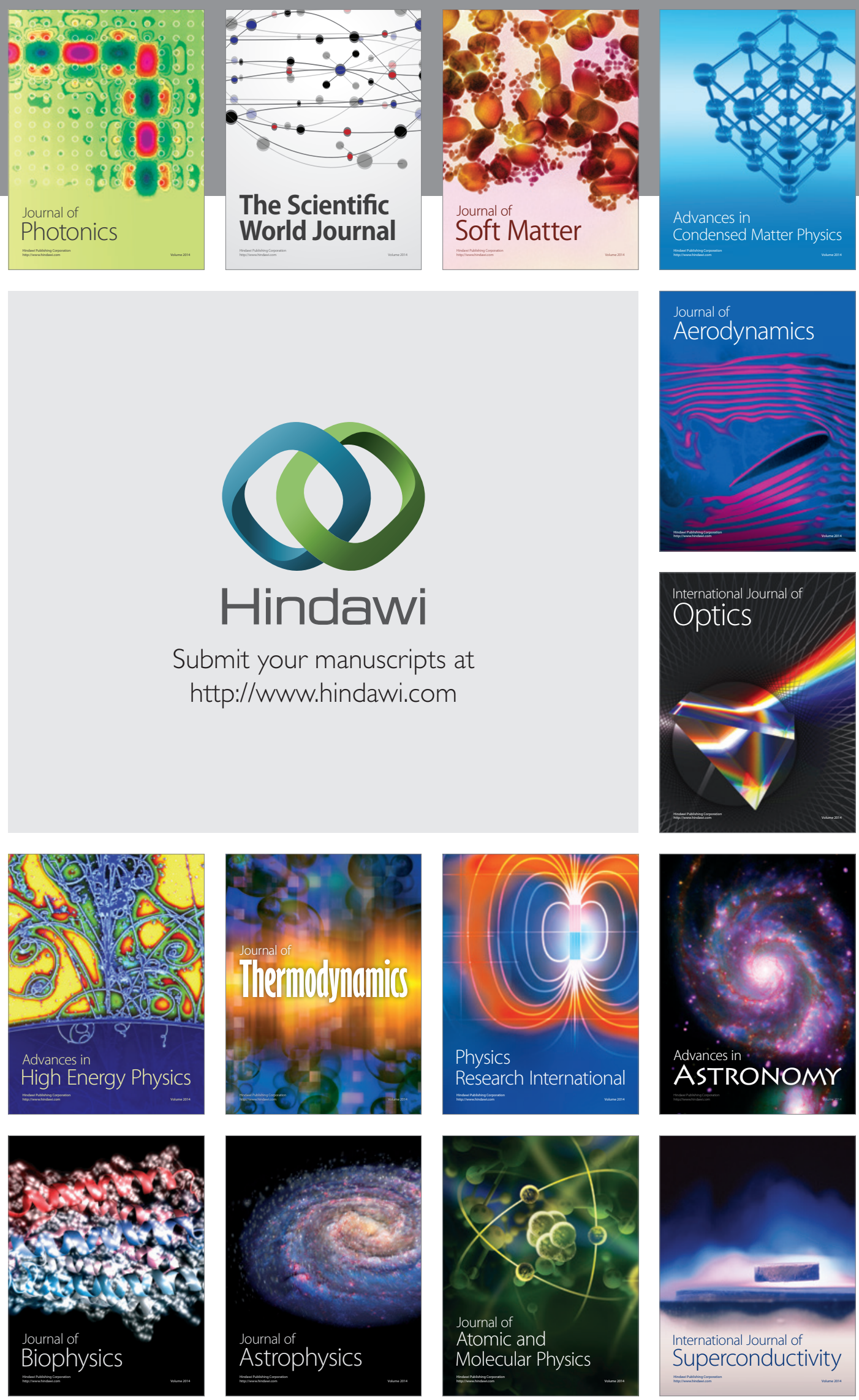
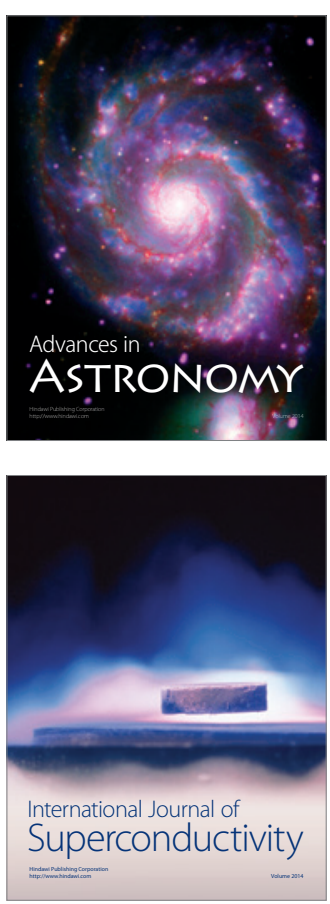